\title{
A new magnetic resonance imaging approach for discriminating Sardinian sheep milk cheese made from heat-treated or raw milk
}

\author{
G. Mulas, T. Roggio, S. Uzzau, and R. Anedda ${ }^{1}$ \\ Porto Conte Ricerche S.r.l., SP 55 Porto Conte/Capo Caccia Km 8.400, Loc Tramariglio 07041 Alghero (SS), Italy
}

\begin{abstract}
The evaluation of milk heat treatment on dairy products via reliable analytical methods is a challenging issue that involves both industrial and fundamental research. We describe a new magnetic resonance imaging (MRI) protocol for discriminating Sardinian sheep milk cheese originating from heat-treated or raw milk. Thirty-six samples (18 pecorino cheeses manufactured from heattreated milk and 18 Fiore Sardo cheeses made from raw milk) were investigated by means of MRI and bi-exponential signal decay analysis. The protocol is capable of discerning cheeses by virtue of the different distribution of the transversal $\left(T_{2}\right)$ relaxation time constant. Cheeses from heat-treated milk showed a significantly higher area fraction $(\approx 70-80 \%)$, corresponding to the fast relaxing water protons $\left(T_{2} \approx 9 \mathrm{~ms}\right)$, compared with raw milk cheeses, whereas the opposite was observed for the long $T_{2}\left(T_{2} \approx 35 \mathrm{~ms}\right)$ proton population. The MRI protocol described is rapid and nondestructive, and it provides statistically significant discrimination between ewe milk cheeses made from heat-treated and raw milk.
\end{abstract}

Key words: thermized milk, raw milk, Fiore Sardo protected designation of origin, magnetic resonance imaging (MRI)

\section{INTRODUCTION}

The need to objectively recognize cheeses made from raw and heat-treated milk is shared by many cheese producers. Parmigiano Reggiano, Stracchino, Taleggio, Asiago d'Allevo (all from Italy), Sao Jorge (Azores), Abondance, Brie, Camembert (all from France), and Boereenkas (the Netherlands) are just few examples of cheese manufactured starting from raw milk.

Despite a number of studies, discriminating between dairy products manufactured from raw and heat-treated

Received January 22, 2013.

Accepted August 24, 2013.

${ }^{1}$ Corresponding author: anedda@portocontericerche.it milk remains a challenging analytical task that attracts great interest (Hirvi and Griffiths, 1998; Pinto et al., 2012). One reason for this interest is the fact that some cheese factories, disregarding traditional recipes and production protocol specifications, submit raw milk to thermization or pasteurization [hereafter, heat-treated (HT) milk], even for the manufacture of cheeses that should be produced from raw milk. Heat treatment of milk may have several advantages, such as safeguarding the hygiene of industrial production pipelines, preventing the appearance of possible off-flavors, ensuring longer shelf life, increasing product safety, and increasing cheese yield (Kelly et al., 2006).

The use of raw milk is one of the most relevant characteristics of the typical Sardinian cheese Fiore Sardo. Other Sardinian sheep milk cheeses, generally referred to as pecorino, can be produced from HT milk. In fact, in modern dairy technology, ewe milk intended for pecorino cheese manufacture is generally subjected to thermization $\left(64-68^{\circ} \mathrm{C}\right.$ for $\left.15 \mathrm{~s}\right)$ or pasteurization $\left(72^{\circ} \mathrm{C}\right.$ for $15 \mathrm{~s}$ ).

Fiore Sardo is a hard cheese produced from raw whole ewe milk. It is one of the oldest-known Mediterranean cheeses, dating back to the Bronze Age, and obtained Protected Designation of Origin status from the European Commission in 1996 (EC Regulation no. 1263/96).

Fiore Sardo cheese is primarily made on artisanal farms according to the following cheesemaking protocol: milk is curdled using lamb rennet paste at 34 to $36^{\circ} \mathrm{C}$, and then the curd is cut and left to drain. As soon as the rinds are formed, they are superficially treated with warm water or scotta (a by-product of ricotta cheese production) to help develop a thick outer rind, and salted. Cheese maturation includes a smoking treatment for $2 \mathrm{wk}$ at 18 to $20^{\circ} \mathrm{C}$, ripening in a cellar at 10 to $15^{\circ} \mathrm{C}$, and finally the treatment of the rinds with an emulsion of olive oil, wine vinegar, and salt.

Currently, the Consortium for the Protection of Fiore Sardo Cheese (CPFSC; Consorzio per la Tutela del Formaggio Fiore Sardo) guarantees the safeguard of the original cheesemaking protocol and it aims to support 
and promote the commercialization of the original typical product. Monitoring milk quality is mandatory to reach these goals.

Although the influence of heat treatment on the microbiological quality and composition of raw milk has been investigated in recent years (Little et al., 2008; Samelis et al., 2009), a scientifically sound analytical method that would enable rapid discrimination of cheese produced from raw or HT milk is still lacking. Therefore, finding a reliable, nondestructive, and rapid analytical method would be valuable for both scientific and technological reasons.

Magnetic resonance imaging (MRI) is a powerful imaging technique widely used in clinical medicine (Caravan, 2006). It is one of the most popular imaging tools because of its noninvasiveness and excellent resolution. Magnetic resonance imaging contrast depends on the physicochemical properties of the system, such as relaxation times $\left(T_{1}\right.$ or $\left.T_{2}\right)$, proton density, and diffusion (Ramos-Cabrer et al., 2006), which makes it particularly suitable for food analysis. In fact, a wide range of nuclear magnetic resonance (NMR) and MRI applications are described in the food science and dairy science literature (see review by Mariette, 2006a,b), such as micro-imaging of the curd granules and joint structure of Emmentaler (Mahdjoub et al., 2003), or evaluating the effects of freezing on Mozzarella (Kuo et al., 2003).

The heterogeneity in the chemical composition of cheese leads to difficulties in the interpretation of magnetic resonance relaxation data. Cheese is a complex mixture of molecular components, where fat, water, salt and minerals are contained within a protein (mainly casein) network (Everett and Olson, 2003; Everett and Auty, 2008).

It is well established that water molecules can be found in cheese in a variety of different environments, causing a spectrum of spin-spin proton relaxation times (Budiman et al., 2002). Therefore, proton magnetic resonance signal decay is multi-exponential in cheese, because of the multiple contributions originating from nuclei of different components (water, fat, proteins) in different environments.

Several methods have been developed in the attempt to reduce the complexity of magnetic resonance analysis on dairy products; for example, separately assessing water and fat in cheese (Castell-Palou et al., 2012). A valuable MRI approach is the chemical shift selective (CHESS) suppression technique, based on the difference in resonance frequency between fat and water protons (Ruan et al., 1998). Additionally, suitable software programs are now available that are specifically designed for magnetic resonance multi-exponential decay image analysis, which allows easy quantification of the sub-voxel structure due to water compartmentalization (Bjarnason and Mitchell, 2010).

In this scenario, we explored the possibility of developing an MRI protocol designed to discriminate between Sardinian cheese made from raw milk and that made from HT milk. We demonstrate for the first time, to the best of our knowledge, a feasible, rapid, and nondestructive method.

\section{MATERIALS AND METHODS}

\section{Samples}

A total of 30 commercial Sardinian sheep milk cheese samples (bought at local markets or directly from producers) were analyzed. Of these, 15 were produced from HT sheep milk (pecorino) and 15 from raw whole sheep milk (Fiore Sardo PDO cheese).

The ripening stage (between 4 and $6 \mathrm{mo}$ ), fat content of the DM (minimum of 35\%), and moisture content (MC) were similar for all samples. Both Fiore Sardo PDO and pecorino cheeses were made from uncooked curd. Because the commercial cheeses were manufactured by different producers and from different milk, minimal predictable differences were expected. Therefore, 6 additional cheese samples were prepared by the CPFSC following the technical policies for production [European Community Reg. no. 1263, July 1st, 1996] and using the same milk. Three samples (R1, R2, and R3) were made from raw milk (as required by the original recipe), and 3 samples (T1, T2, T3) were produced from the same milk after thermization $\left(68^{\circ} \mathrm{C}\right.$ for $\left.15 \mathrm{~s}\right)$. All analytical samples, cut from the center of the cheese wheel, were stored at $4^{\circ} \mathrm{C}$ until MRI analysis.

\section{Moisture Content and Lipid Content}

Moisture content was measured according to AOAC International (2000) method 33.7.03. Lipid extracts were obtained following the method of Folch et al. (1957).

\section{MRI Measurements}

All samples were equilibrated in a cold room at $20^{\circ} \mathrm{C}$ for $1 \mathrm{~h}$ before MRI analysis. The temperature was checked before and immediately after MRI measurements by inserting a thermocouple into the cheese paste; no deviation from the temperature of $20^{\circ} \mathrm{C}$ was measured. The MRI experiments were performed using a Bruker Avance $300 \mathrm{MHz}$ equipped with a microimaging Micro2.5 probe (Bruker Biospin, Karlsruhe, Germany). A conventional Carr Purcell Meiboom Gill (CPMG) spin echo sequence was used: single slice; echo time 
$(\mathbf{T E})=7.907 \mathrm{~ms} ;$ repetition time $(\mathbf{T R})=3 \mathrm{~s} ;$ echoes $=$ $64 ;$ matrix $=128 \times 128 ;$ number of excitations $=1-3$; acquisition time $=6-18 \mathrm{~min}$. The MRI experiments were performed in duplicate for each sample.

To selectively analyze signals from fat or water, a protocol was optimized that took advantage of the dissimilarity in the chemical shifts of the 2 molecular classes. Water suppression was achieved by using a preparatory step consisting of a spectrally selective pulse (see Supplementary Data for details: http://dx.doi. org/10.3168/jds.2013-6607) centered on water proton resonance. Similarly, the fat signal was cancelled with a $90^{\circ}$ selective pulse applied before the conventional CPMG spin echo sequence at a frequency offset of 3.5 ppm with respect to water (preparatory fat saturation scheme provided by Bruker Topspin). A CPMG spin echo sequence without the saturation scheme $(\mathrm{TE}=$ $7.907 \mathrm{~ms}, \mathrm{TR}=3 \mathrm{~s}$, matrix $=128 \times 128)$ was always acquired as control.

\section{Multi-Exponential Decay Image Analysis}

The AnalyzeNNLS software package was used to deconvolute the multi-exponential transversal relaxation time $\left(\boldsymbol{T}_{2}\right)$ decay of water and fat proton signals. Bruker MRI image data were converted into multiecho image data (MEID) files by means of Matlab software (version R2013b; Math Works Inc., Natick, MA). The $T_{2}$ distribution graphs were first created from manually drawn regions of interest, and then the geometric mean $T_{2}$ ( $T_{2}$ relaxation time constant on a logarithmic scale) and the area fraction (obtained by dividing the sum of the $T_{2}$ distribution within a desired region between $T_{2 \min }$ and $T_{2 \max }$ by the sum of all $T_{2}$ distributions) were calculated for all samples.

\section{Statistical Analysis}

The results are expressed as means \pm standard error of the mean. Statistical analysis was carried out using the GraphPad Prism software (version 5.03, GraphPad software Inc., San Diego, CA). Two-way ANOVA and $t$ test were used to determine differences between cheeses made from raw and HT milk. A $P$-value $<0.05$ was considered statistically significant.

\section{RESULTS AND DISCUSSION}

\section{Visual Inspection and MRI Images}

Figure 1 shows $T_{2}$ weighted images of cheese samples made from HT milk (Figure 1a) and from raw milk (Figure 1b), acquired with a standard CPMG spin echo sequence without saturation. The images, reported
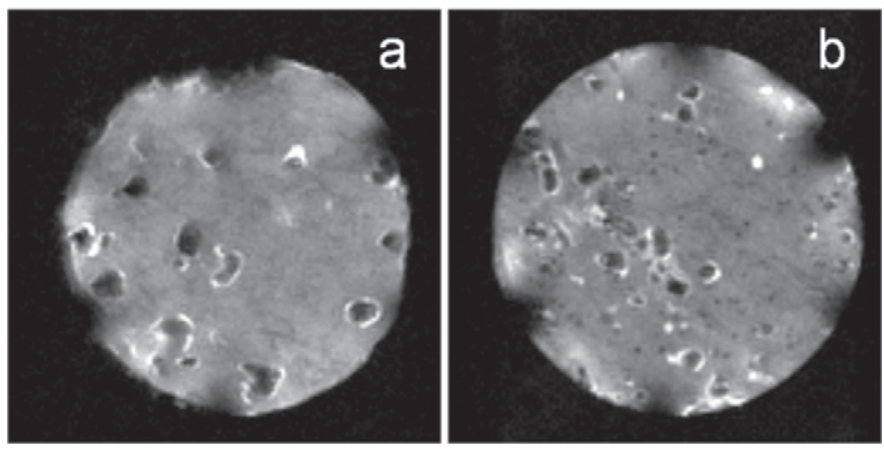

Figure 1. Transversal relaxation time constant $\left(T_{2}\right)$ weighted axial images acquired with a standard Carr Purcell Meiboom Gill (CPMG) spin echo sequence: (a) Cheese made from heat-treated milk, and (b) cheese made from raw milk. The chemical shift artifact is present because images were acquired without fat saturation.

as representative examples of what is generally observed, illustrate that the raw milk cheese showed a higher number of small and approximately round holes ("eyes") compared with the HT milk cheese. The HT milk cheese exhibited a more uniform paste with fewer slits, cracks, or holes compared with its raw milk counterpart. Moreover, the eyes in HT cheeses were larger on average. Cheese eyes are formed as a consequence of fermentative processes induced by cheese microorganisms; therefore, it is generally believed that heat treatment of milk hinders the formation of eyes (Salvadori del Prato, 1998; Polychroniadou, 2001).

The distinction between raw milk Fiore Sardo PDO and HT milk pecorino has, to date, been carried out by visually inspecting the cheese samples. Although only a few published scientific reports exist on the topic (Bàrcenas et al., 2007; Scintu et al., 2010), according to Sardinian producers of Fiore Sardo PDO and skilled cheese tasters, Fiore Sardo PDO (i.e., made from raw milk) can be discriminated from HT counterparts by inspection of the paste, evaluating its texture and color and assessing different cheese eyes (Piga et al., 2012).

From a scientific perspective, the sensory attributes of floury and grainy, together with firmness, crumbliness, and friability, are associated with Fiore Sardo PDO cheese texture. Such textural characteristics are believed to be affected by heat treatment of milk (Mendia et al., 1999; Buffa et al., 2001; Gonzalez Viñas et al., 2007; Scintu et al., 2010; Morales-Celaya et al., 2012). However, identifying such sensorial differences may be time consuming because it requires cheese sampling and trained expert panelists.

In this regard, MRI offers an advantage because it provides information on cheese microstructure in a rapid and nondestructive way and with high spatially resolved resolution. In addition to enabling assessment og paste macrostructure, MRI provides specific physi- 
cochemical parameters associated with cheese molecular structure.

For example, MRI has been exploited to observe the curd granules and joint structure, to visualize cheese eyes in intact wheels (Mahdjoub et al., 2003), and to elicit more objective parameters such as relaxation times or proton density (Mariette, 2006b).

Regarding Fiore Sardo images acquired for the present study, it should be noted that differences in eye development between raw milk and HT milk cheeses are not always evident on first inspection of MRI images. These observations may easily lead to sample misclassifications if not verified by quantifying the differences on a large number of samples and performing robust statistical analyses. Such a statistical examination of MRI images is beyond the scope of the present work. To find rapid procedures that could be easily implemented to discriminate Fiore Sardo PDO from its imitations, a more in-depth study of the interactions between the molecular components of cheese provides a clear discrimination between the 2 cheeses.

\section{$T_{2}$ Populations of Water and Fat Protons}

Representative $T_{2}$ distributions of water protons (obtained from MRI images acquired with fat suppression) are reported in Figure 2 for HT (Figure 2a) and raw milk (Figure $2 \mathrm{~b}$ ) cheeses. Two main $T_{2}$ populations (peaks in Figure 2) were detected for all cheese samples, whereas a third small peak on the right was due to noise (Supplementary Data: http://dx.doi. org/10.3168/jds.2013-6607).

The 2 principal peaks detected were centered approximately at $9 \mathrm{~ms}$ (hereafter, the first population, $\mathbf{1 P}$ ) and at $35 \mathrm{~ms}$ (second population, 2P). Each peak was characterized by an area fraction and a geometric mean $T_{2}$ value (see Materials and Methods). Data related to commercial samples are shown in Table 1.

Geometric mean $T_{2}$ values corresponding to the 2 populations were not significantly influenced by heat treatment of milk, being approximately the same for HT and raw milk cheeses (Table 1). On the other hand, area fractions were significantly different for $\mathrm{HT}$ and raw milk cheeses. Cheeses made from HT milk consistently exhibited a higher mean area fraction for $1 \mathrm{P}$ $(0.78 \pm 0.05)$ than did raw milk cheeses $(0.39 \pm 0.14)$. The opposite was found for $2 \mathrm{P}$ - an area fraction value of $0.19 \pm 0.05$ was observed for cheeses made from HT milk, whereas a value of $0.56 \pm 0.14$ was obtained for raw milk cheeses (Table 1 ).

The MRI analyses on CPFSC samples confirmed the trend observed for commercial cheeses (Table 2). The mean area fraction relative to $1 \mathrm{P}$ was significantly higher for CPFSC HT milk cheeses $(0.74 \pm 0.04)$ com-
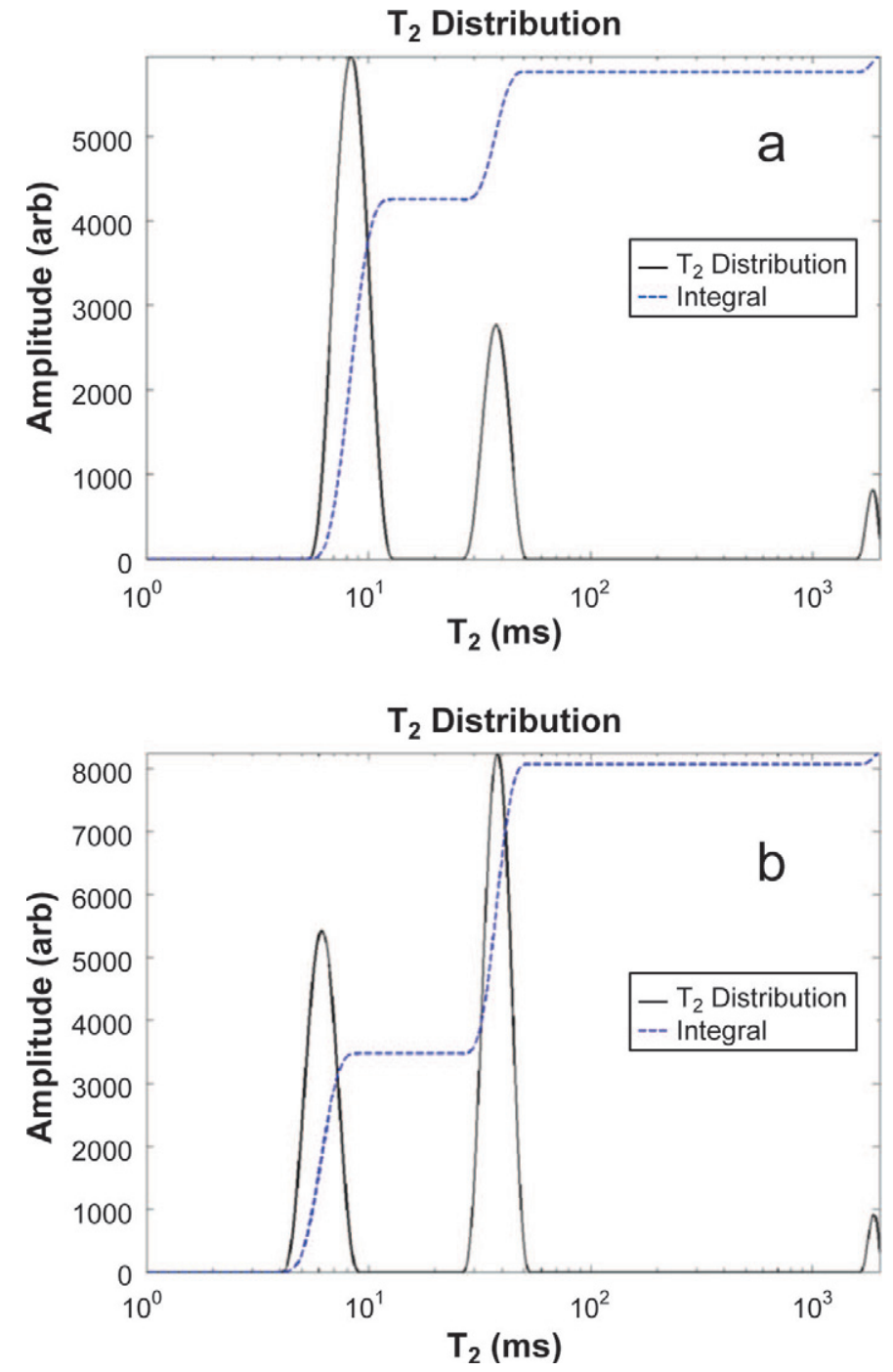

Figure 2. Water transversal relaxation time constant $\left(T_{2}\right)$ distribution and cumulative sum graphs: (a) $T_{2}$ distribution of a heat-treated milk cheese; (b) $T_{2}$ distribution of a raw milk cheese. Color version available in the online PDF.

pared with CPFSC raw milk cheeses $(0.51 \pm 0.09)$. The differences observed and graphically depicted in Figure 3 were statistically significant $(P<0.0001$ for commercial cheese; $P<0.05$ for CPFSC samples).

No significant differences between HT and raw milk cheese were shown by analyzing $T_{2}$ distributions of fat protons after water signal suppression. Data relative to CPFSC samples are shown, as an example, in Table 3 and in Figure 4. All cheese samples analyzed exhibited only one $T_{2}$ population relative to fat protons. Geometric mean $T_{2}$ values of fat protons were in the range of the $2 \mathrm{P}$ population of water protons, although they were characterized by a generally broader distribution (20-100 ms), as was expected (Chaland et al., 2000; Mariette, 2006a,b). Therefore, we focused on $T_{2}$ 
Table 1. Mean $( \pm \mathrm{SEM})$ geometric transversal relaxation time constant $T_{2}$ and area fraction $(\mathrm{AF})$ of water protons for heat-treated or raw milk commercial cheeses ${ }^{1}$

\begin{tabular}{|c|c|c|c|c|}
\hline \multirow[b]{2}{*}{ Sample } & \multicolumn{2}{|c|}{ 1st population } & \multicolumn{2}{|c|}{ 2nd population } \\
\hline & $T_{2}(\mathrm{~ms})$ & $\mathrm{AF}$ & $T_{2}(\mathrm{~ms})$ & $\mathrm{AF}$ \\
\hline \multicolumn{5}{|c|}{ Heat-treated } \\
\hline 1 & $7.02 \pm 0.36$ & $0.72 \pm 0.05$ & $28.40 \pm 2.20$ & $0.24 \pm 0.06$ \\
\hline 2 & $7.14 \pm 0.36$ & $0.68 \pm 0.01$ & $29.90 \pm 0.04$ & $0.27 \pm 0.01$ \\
\hline 3 & $7.84 \pm 0.34$ & $0.81 \pm 0.02$ & $24.62 \pm 0.88$ & $0.15 \pm 0.01$ \\
\hline 4 & $7.50 \pm 0.14$ & $0.79 \pm 0.04$ & $26.39 \pm 1.69$ & $0.17 \pm 0.03$ \\
\hline 5 & $6.45 \pm 0.01$ & $0.70 \pm 0.02$ & $24.96 \pm 2.09$ & $0.26 \pm 0.01$ \\
\hline 6 & $8.58 \pm 0.32$ & $0.76 \pm 0.04$ & $29.82 \pm 1.77$ & $0.19 \pm 0.03$ \\
\hline 7 & $7.77 \pm 0.23$ & $0.72 \pm 0.01$ & $27.65 \pm 2.13$ & $0.23 \pm 0.01$ \\
\hline 8 & $9.85 \pm 0.11$ & $0.79 \pm 0.01$ & $28.79 \pm 0.99$ & $0.17 \pm 0.02$ \\
\hline 9 & $9.44 \pm 0.39$ & $0.83 \pm 0.02$ & $27.50 \pm 1.41$ & $0.15 \pm 0.02$ \\
\hline 10 & $10.59 \pm 0.16$ & $0.83 \pm 0.03$ & $31.68 \pm 0.95$ & $0.13 \pm 0.03$ \\
\hline 11 & $9.98 \pm 0.24$ & $0.80 \pm 0.01$ & $40.28 \pm 0.42$ & $0.16 \pm 0.01$ \\
\hline 12 & $9.06 \pm 0.11$ & $0.81 \pm 0.04$ & $27.18 \pm 1.84$ & $0.15 \pm 0.02$ \\
\hline 13 & $9.23 \pm 0.34$ & $0.84 \pm 0.03$ & $35.44 \pm 0.91$ & $0.13 \pm 0.01$ \\
\hline 14 & $7.98 \pm 0.24$ & $0.83 \pm 0.01$ & $29.47 \pm 1.70$ & $0.14 \pm 0.01$ \\
\hline 15 & $8.27 \pm 0.26$ & $0.72 \pm 0.04$ & $26.93 \pm 1.40$ & $0.24 \pm 0.04$ \\
\hline Mean & 8.45 & $0.78^{\mathrm{a}}$ & 29.27 & $0.19^{\mathrm{c}}$ \\
\hline SD & 1.21 & 0.05 & 4.06 & 0.05 \\
\hline \multicolumn{5}{|l|}{ Raw } \\
\hline 16 & $6.38 \pm 0.49$ & $0.51 \pm 0.01$ & $32.36 \pm 1.71$ & $0.43 \pm 0.01$ \\
\hline 17 & $8.14 \pm 1.35$ & $0.52 \pm 0.07$ & $40.26 \pm 4.78$ & $0.44 \pm 0.09$ \\
\hline 18 & $7.75 \pm 1.43$ & $0.39 \pm 0.02$ & $34.70 \pm 3.28$ & $0.55 \pm 0.03$ \\
\hline 19 & $6.99 \pm 0.85$ & $0.36 \pm 0.06$ & $38.00 \pm 0.03$ & $0.61 \pm 0.06$ \\
\hline 20 & $13.91 \pm 6.11$ & $0.23 \pm 0.03$ & $35.42 \pm 0.27$ & $0.74 \pm 0.03$ \\
\hline 21 & $9.42 \pm 0.01$ & $0.43 \pm 0.13$ & $38.92 \pm 2.88$ & $0.53 \pm 0.13$ \\
\hline 22 & $9.46 \pm 0.17$ & $0.23 \pm 0.04$ & $34.82 \pm 2.08$ & $0.75 \pm 0.03$ \\
\hline 23 & $7.86 \pm 1.06$ & $0.20 \pm 0.05$ & $33.58 \pm 1.80$ & $0.76 \pm 0.04$ \\
\hline 24 & $8.10 \pm 0.51$ & $0.62 \pm 0.01$ & $31.05 \pm 1.24$ & $0.34 \pm 0.01$ \\
\hline 25 & $6.57 \pm 0.46$ & $0.40 \pm 0.04$ & $32.11 \pm 0.63$ & $0.57 \pm 0.04$ \\
\hline 26 & $7.09 \pm 1.73$ & $0.45 \pm 0.06$ & $28.17 \pm 2.26$ & $0.49 \pm 0.06$ \\
\hline 27 & $11.06 \pm 1.10$ & $0.54 \pm 0.02$ & $50.78 \pm 0.87$ & $0.41 \pm 0.04$ \\
\hline 28 & $11.13 \pm 2.40$ & $0.54 \pm 0.04$ & $50.12 \pm 3.18$ & $0.41 \pm 0.04$ \\
\hline 29 & $8.95 \pm 1.91$ & $0.29 \pm 0.09$ & $38.70 \pm 0.00$ & $0.67 \pm 0.09$ \\
\hline 30 & $9.27 \pm 1.94$ & $0.21 \pm 0.09$ & $37.08 \pm 2.47$ & $0.76 \pm 0.01$ \\
\hline Mean & 8.81 & $0.39^{\mathrm{b}}$ & 37.07 & $0.56^{\mathrm{d}}$ \\
\hline SD & 2.03 & 0.14 & 6.34 & 0.14 \\
\hline
\end{tabular}

${ }^{\mathrm{a}-\mathrm{d}}$ Mean values with different superscripts are significantly different $(P<0.0001)$.

${ }^{1}$ Measurements were performed in duplicate. Samples were numbered consecutively from 1 to 30 , with samples 1-15 representing heat-treated milk cheeses and samples 16-30 cheeses made from raw milk. 1st population and 2nd population correspond to water protons with short $T_{2}$ and long $T_{2}$, respectively.

populations of water protons, deferring a more careful $T_{2}$ analysis of fat protons to additional specific experiments.

The reliability of the $2 T_{2}$ components $1 \mathrm{P}$ and $2 \mathrm{P}$ was sustained and validated by 2 considerations: first, the results here presented are in good agreement with several published papers in which proton relaxation in cheese and cheese analogs has been analyzed by means of low-resolution time-domain NMR (taking into account some expected minor dissimilarities due to different temperatures of analysis and to dissimilar cheesemaking processes; Chaland et al., 2000; Gianferri et al., 2007a,b; Noronha et al., 2008); second, our results are consistent with previous results and guidelines published on multi-exponential relaxation behavior of water in other heterogeneous systems, especially in the biomedical and clinical applications of MRI (Graham et al., 1996; Fenrich et al., 2001).

Chaland et al. (2000), for example, provided a complete description of the effects of cheese composition on proton magnetic relaxation. In particular, they characterized fat and water states in soft and hard cheeses by means of ${ }^{1} \mathrm{H}$ NMR relaxometry. The transverse (spinspin) relaxation behavior of the protons in cheeses was described by 4 relaxation components: compsol 1 and compsol 2, representing the solid part; and compliq 1 and compliq2, constituting the liquid part. They found that the intensity of the solid part was mainly explained by crystalline fat, whereas the liquid part was mostly influenced by water $\left(T_{2}\right.$ compliq $1 \approx 10 \mathrm{~ms} ; T_{2}$ compliq $2 \approx 36 \mathrm{~ms}$ ), with slight differences between hard and soft cheese. 
Table 2. Mean ( \pm SEM) geometric transversal relaxation time constant $T_{2}$ and area fraction (AF) of water protons and for heat-treated or raw milk CPFSC (Consortium for the Protection of Fiore Sardo Cheese) cheeses $^{1}$

\begin{tabular}{lccccc}
\hline & \multicolumn{2}{c}{ 1st population $^{2}$} & & \multicolumn{2}{c}{ 2nd population } \\
\cline { 2 - 3 } \cline { 5 - 6 } Sample & $T_{2}(\mathrm{~ms})$ & $\mathrm{AF}$ & & $T_{2}(\mathrm{~ms})$ & $\mathrm{AF}$ \\
\hline $\mathrm{T} 1$ & $8.75 \pm 0.02$ & $0.71 \pm 0.01$ & & $37.57 \pm 0.12$ & $0.26 \pm 0.01$ \\
T2 & $7.38 \pm 0.08$ & $0.75 \pm 0.01$ & & $32.83 \pm 0.15$ & $0.23 \pm 0.00$ \\
T3 & $8.15 \pm 0.08$ & $0.78 \pm 0.01$ & & $32.98 \pm 0.50$ & $0.19 \pm 0.01$ \\
Mean & 8.09 & $0.74^{\mathrm{a}}$ & & 34.46 & $0.22^{\mathrm{c}}$ \\
SD & 0.69 & 0.04 & & 3.70 & 0.04 \\
R1 & $10.48 \pm 1.48$ & $0.41 \pm 0.02$ & & $35.77 \pm 1.66$ & $0.52 \pm 0.01$ \\
R2 & $7.54 \pm 0.10$ & $0.53 \pm 0.00$ & & $36.81 \pm 0.55$ & $0.42 \pm 0.00$ \\
R3 & $8.01 \pm 1.21$ & $0.59 \pm 0.03$ & & $38.64 \pm 3.12$ & $0.37 \pm 0.02$ \\
Mean & 8.67 & $0.51^{\mathrm{b}}$ & & 37.07 & $0.44^{\mathrm{d}}$ \\
SD & 1.58 & 0.09 & & 1.45 & 0.08 \\
\hline
\end{tabular}

${ }^{\mathrm{a} d}$ Means within a column with different superscripts are significantly different $(P<0.05)$.

${ }^{1}$ Measurements were performed in duplicate. T1, T2, and T3 represent samples from 3 different heat-treated milk cheese wheels; samples R1, R2, and R3 refer to 3 cheese wheels made from raw milk, with mean values and standard deviations for each group. All CPFSC cheese wheels were manufactured from the same milk but subjected to different heat treatments.

${ }^{2} 1$ st population and 2 nd population correspond to water protons with short $T_{2}$ and long $T_{2}$, respectively.

Gianferri et al. (2007a,b) studied Mozzarella di Bufala Campana by means of proton NMR transverse relaxation measurements and found 3 different water populations, namely "serum" (or bulk) water $\left(T_{2}=\right.$ 480-950 ms), "entrapped" water inside the mesh of the casein network $\left(T_{2}=16-47 \mathrm{~ms}\right)$, and "water in junction zones" $\left(T_{2}=7 \mathrm{~ms}\right)$. Those authors hypothesized the existence of water in an interstitial form within the narrow meshes of junction zones, the short transverse relaxation time of which $\left(T_{2}=7 \mathrm{~ms}\right)$ was dominated by chemical exchange with protein protons.

As stated above, the observation of bi-exponential $T_{2}$ relaxation in MRI images of cheeses also has a more logical physicochemical explanation in light of previous biomedical NMR-MRI studies on proton transverse relaxation in heterogeneous systems. Such studies demonstrated the usefulness of MRI in resolving multiexponential features of proton magnetization decay and especially investigated the instrumental details and fitting algorithms to face the principal limitations of MRI; that is, the long echo times and the low signal-to-noise ratio (Fenrich et al., 2001). Briefly, in an MRI $T_{2}$ study, optimization of parameters, such as echo time (TE) and signal-to-noise ratio, is essential for the reliable elaboration of multi-exponential $T_{2}$ decay curves. In this regard, previous MRI studies, mostly directed to clinical MRI applications, confirmed that to perform quantitative analysis of multicomponent $T_{2}$ relaxation data, high signal-to-noise ratios $(\geq 100)$ are required (Laule et al., 2007). In the case of in vivo imaging, because of hardware constraints and safety aspects, typical MRI parameters are echo spacings in the range $10 \mathrm{~ms}<\mathrm{TE}<15 \mathrm{~ms}$ and signal-to-noise ratio values of 100 or lower. Despite these limitations, it has been possible to successfully quantify in vivo the short $T_{2}$ component of myelin (10 ms $<T_{2}<15 \mathrm{~ms}$ ) from human white matter, both in terms of mean $T_{2}$ values and water content (MacKay et al., 1994; Whittall et al., 1997). These pioneering findings indicated that MRI is a powerful diagnostic tool for quantitative measure of myelin loss in demyelinating diseases (Fenrich et al., 2001; McCreary et al., 2009) and paved the way for new applications of MRI for $T_{2}$ relaxation studies in other multicomponent heterogeneous systems. Many studies have been published since the mid-1990s to specifically address this issue (Does and Snyder, 1995; Anastasiou and Hall, 2004; Dula et al., 2009). The same approach, in which NMR and MRI $\left(\mathrm{TE}=6 \mathrm{~ms}, 6 \mathrm{~ms}<T_{2}<10\right.$ $\mathrm{ms}$ ) have been combined to describe the same system, has been applied to cheese characterization (Kuo et al., 2001, 2003).

As in the discussion above, the first population of water proton $T_{2}\left(1 \mathrm{P}, 7 \mathrm{~ms}<T_{2}<14 \mathrm{~ms}\right)$ found in the current study can be considered a real component of relaxation and not an artifact, even if acquired with a TE of the same magnitude of $T_{2}$ values themselves. In fact, the signal-to-noise ratio of cheese images (ranging from 110 to 150) presented here should be sufficient to characterize the short $T_{2}$ with only a few echoes. To further confirm this result, and following the guidelines indicated by previous investigations, we acquired images of cheese with signal-to-noise ratios as high as 515 (at expense of acquisition time), observing that the results ( $T_{2}$ and area fractions) did not appreciably differ from results reported in Tables 1 and 2 .

Interestingly, consistent results (i.e., the similar $T_{2}$ distribution trend) revealed for the 30 commercial samples and the 6 CPFSC cheeses suggest that heat 

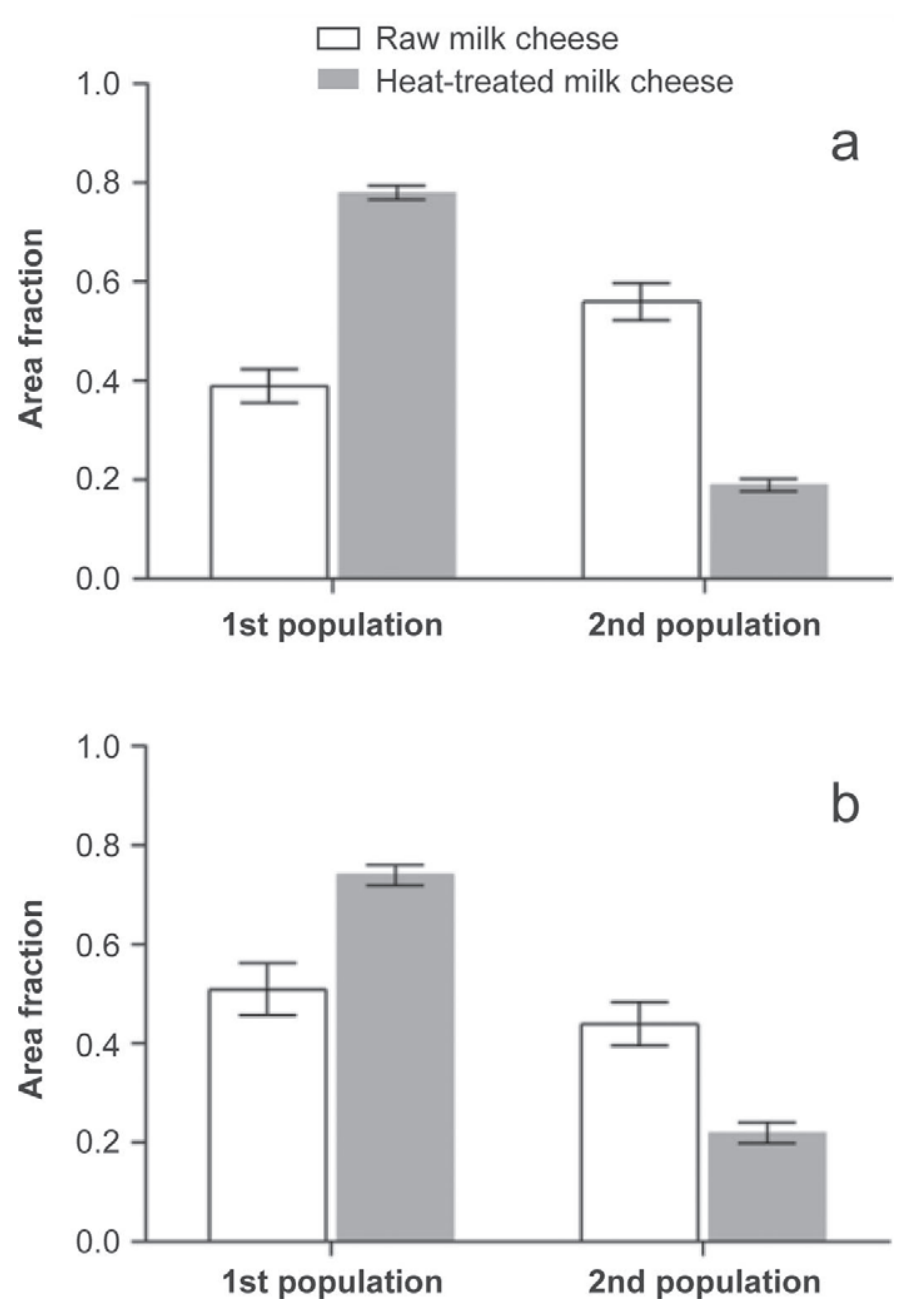

Figure 3. Transversal relaxation time constant $\left(T_{2}\right)$ area fraction histograms with mean and standard error bars of heat-treated and raw milk cheeses: (a) $T_{2}$ area fraction of commercial cheeses; (b) $T_{2}$ area fraction of Consorzio per la Tutela del Formaggio Fiore Sardo cheeses.

treatment of milk is the major source of product differentiation, which therefore assumes a role of dominance over other variables (e.g., different production areas, milk composition, and cheesemaking procedures). Consequently, considering that the most significant changes induced by heat treatments on milk constituents are those that involve proteins (Kelly et al., 2006), the different $T_{2}$ distribution exhibited by HT (pecorino) and raw milk (Fiore Sardo PDO) cheeses could be attributed to a dissimilar interaction between water and protein protons. However, this is just a reasonable hypothesis and additional studies are required to further detail the molecular mechanisms of this multifaceted phenomenon.

The data presented here demonstrate, for the first time, that rapid MRI analyses on intact cheese wheels could provide information on the heat treatment to which the milk has been subjected before the manufacture of Fiore Sardo cheese. The results presented are statistically significant and are not affected by artifacts due to instrumental setting or data-fitting algorithms.

\section{Influence of Fat and Moisture Content on $T_{2}$ Distribution of Water Protons}

Signal suppression, calculated as the ratio of the mean signal intensity with saturation to that measured without saturation, is more efficient for water than for fat $(\approx 6 \%$ and $\approx 18 \%$ residual signal, respectively; Supplementary Figure S1; http://dx.doi.org/10.3168/ jds.2013-6607). Even if relaxation compensation is sometimes exploited to further attenuate fat signal (Ruan et al., 1998), this approach is not suitable for dairy products because the $T_{2}$ of fat is widely dispersed (Mariette, 2006b).
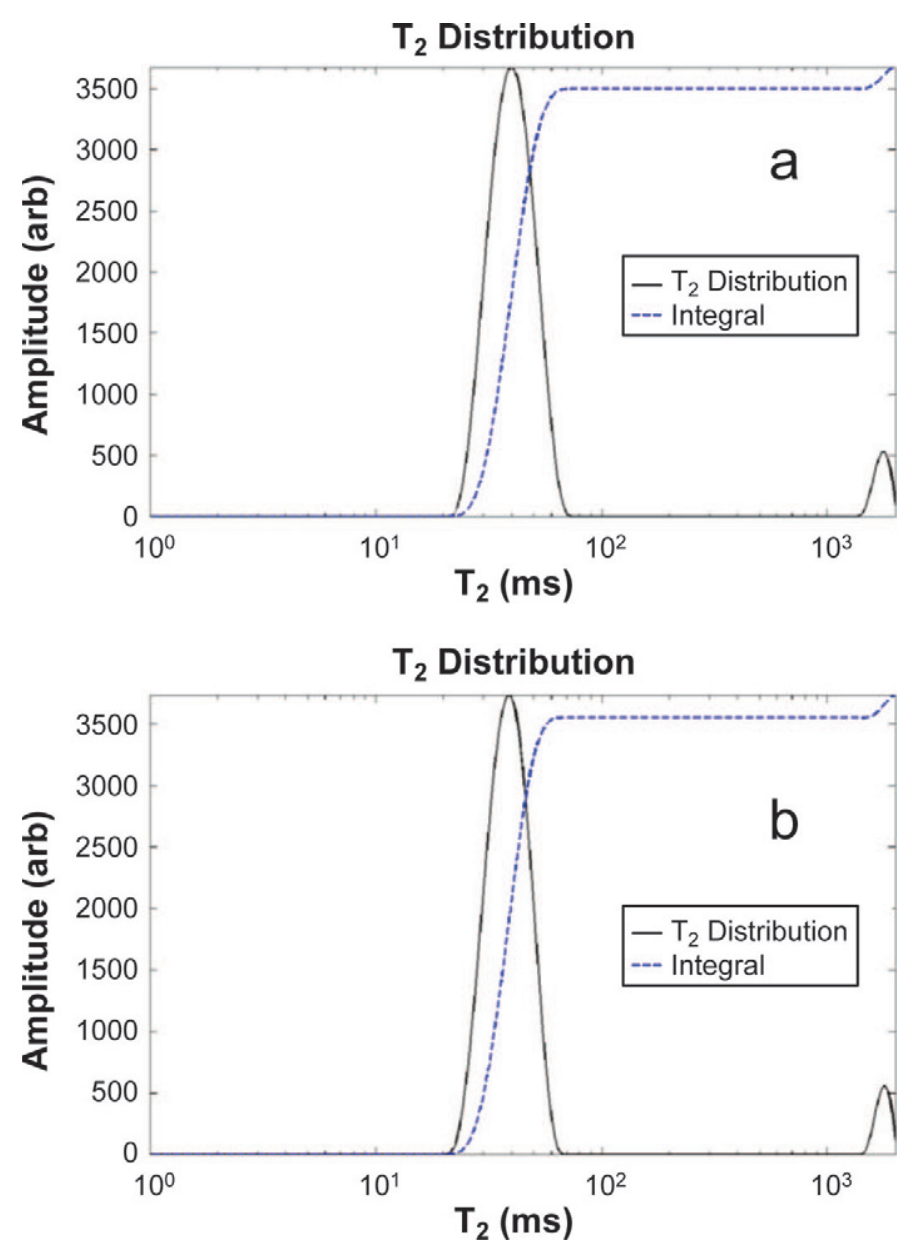

Figure 4. Fat transversal relaxation time constant $\left(T_{2}\right)$ distribution and cumulative sum graphs of Consorzio per la Tutela del Formaggio Fiore Sardo cheeses: (a) $T_{2}$ distribution of a heat-treated milk cheese; (b) $T_{2}$ distribution of a raw milk cheese. Color version available in the online PDF. 
To a first reasonable approximation, the 2 populations of $T_{2}$ observed with fat suppression (Figure 2, Tables 1 and 2) can be directly correlated to different water partitioning within the cheese block, assuming that incomplete fat saturation is considered negligible. In fact, it should be noted that even if most of the fat signal has been suppressed by the specific MRI sequence, the residual fat signal $(\approx 18 \%)$ is superimposed on the $2 \mathrm{P}\left(T_{2} \approx 35 \mathrm{~ms}\right.$; Chaland et al., 2000; Gianferri et al., 2007a). By comparing cheeses with different fat contents, we estimated that this bias was not likely to interfere with the final discriminative power of the technique because the residual fat signal, even for high fat content cheeses, is responsible for only a negligible percentage of the area fraction. As a proof of concept, we analyzed, by fat-suppressed CPMG MRI images, HT milk cheeses containing a higher fat percentage (43.4\% of DM) than their raw milk counterparts $(35 \%$ of $\mathrm{DM}$ ), and their area fraction ratio $1 \mathrm{P}: 2 \mathrm{P}$ was found to be 0.70:0.26, compared with 0.43:0.53 for the raw counterparts.

Moisture content was also measured to investigate its possible influence on results. Cheese samples with similar MC; for example, HT samples 6 and 11 (MC $=29.2 \% \pm 1.9$ and $34.5 \% \pm 0.8$, respectively) and raw milk cheese counterparts 18 and $21(\mathrm{MC}=29.7 \pm 0.4$ and $34.5 \% \pm 0.3$ ), exhibited a completely dissimilar $T_{2}$ distribution (Table 1), indicating that the variance of $T_{2}$ distributions is not influenced by the total amount of water. Therefore, neither water content nor fat content can interfere with the observed $T_{2}$ distributions of water protons.

\section{Multi-Exponential Relaxation, Chemical, and Diffusive Exchange Model}

The observation of 2 relaxation components $\left(1 \mathrm{P} T_{2}\right.$ $\approx 9 \mathrm{~ms} ; 2 \mathrm{P} T_{2} \approx 35 \mathrm{~ms}$ ) in the current study is consistent with a slow diffusive exchange regimen, and the analysis of the dynamics of the system provides useful structural indications of cheese body at the molecular level. The slow diffusive exchange condition is described by Eq. [1] (Hills et al., 1990; Gianferri et al., 2007a):

$$
\left|T_{2,1 \mathrm{P}}^{-1}-T_{2,2 \mathrm{P}}^{-1}\right| \geq \frac{D}{a^{2}}
$$

where $D$ is the water self-diffusion coefficient, $a$ is the characteristic dimension of spatial heterogeneity, and $T_{2,1 \mathrm{P}}^{-1}$ and $T_{2,2 \mathrm{P}}^{-1}$ are the relaxation rates (i.e., inverse of $T_{2}$ ) of water molecules in $1 \mathrm{P}$ and $2 \mathrm{P}$, respectively. Therefore, assuming that, at $20^{\circ} \mathrm{C}, D=1.3 \times 10^{-9}$ $\mathrm{m}^{2} \cdot \mathrm{s}^{-1}$, a typical value observed in dairy products (Mé-
Table 3. Mean $( \pm \mathrm{SEM})$ geometric transversal relaxation time constant $T_{2}$ and area fraction (AF) of fat protons and for heat-treated or raw milk CPFSC (Consortium for the Protection of Fiore Sardo Cheese) cheeses $^{1}$

\begin{tabular}{lcc}
\hline Sample & $T_{2}(\mathrm{~ms})$ & AF \\
\hline T1 & $39.79 \pm 0.04$ & $0.95 \pm 0.01$ \\
T2 & $37.53 \pm 1.64$ & $0.95 \pm 0.01$ \\
T3 & $40.79 \pm 1.28$ & $0.95 \pm 0.00$ \\
Mean & 39.37 & 0.95 \\
SD & 1.67 & 0.00 \\
R1 & $40.65 \pm 2.14$ & $0.95 \pm 0.01$ \\
R2 & $38.47 \pm 1.63$ & $0.95 \pm 0.01$ \\
R3 & $37.58 \pm 0.56$ & $0.96 \pm 0.02$ \\
Mean & 38.90 & 0.95 \\
SD & 1.58 & 0.01 \\
\hline
\end{tabular}

${ }^{1}$ Measurements were performed in duplicate. T1, T2, and T3 represent samples from 3 different heat-treated milk cheese wheels; samples R1, $\mathrm{R} 2$, and R3 refer to 3 cheese wheels made from raw milk, with mean values and standard deviations for each group. All CPFSC cheese wheels were manufactured from the same milk but subjected to different heat treatments.

tais and Mariette, 2003), the criterion for observation of multi-exponential relaxation in heterogeneous systems is met considering that the casein network in Fiore Sardo and pecorino cheeses should have a diffusion distance larger than approximately $4 \mu \mathrm{m}(a \geq 3.8$ $\mu \mathrm{m})$. In addition, a value of diffusive exchange rate constant $k_{\text {diff }}\left(k_{\text {diff }}=\frac{D}{a^{2}}\right)$ of about $80 \mathrm{~s}^{-1}$ can be determined for water molecules (Gianferri et al., 2007a).

The fact that $T_{2,1 \mathrm{P}}^{-1}\left(\approx 113 \mathrm{~s}^{-1}\right)$ is faster than the relaxation rate of the bulk water $\left(T_{2, w}=2.5 \mathrm{~s}\right.$ and $\left.T_{2, w}^{-1}=0.4 \mathrm{~s}^{-1}\right)$ can be explained by considering the fast chemical exchange of water protons with exchangeable protons of protein molecules.

In particular, because the relaxation time of exchangeable protein protons is short $\left(T_{2, \text { prot }} \approx\right.$ tens of $\mu \mathrm{s})$, the relaxation rates are dominated by the exchange term and thus by the fast relaxation time of the exchangeable protein protons according to Eq. [2] (Hills et al., 1989, 1990), expressed below in an abbreviated form (see Supplemental Data for details):

$$
T_{2,1 \mathrm{P}}^{-1}=T_{2, w}^{-1}+P_{\text {prot }} k_{\text {prot }},
$$

where $P_{\text {prot }}$ is the protein-exchangeable protons molar fraction and $k_{\text {prot }}$ is the chemical exchange rate constant between water and protein protons. Assuming that the protein fraction of cheese is composed of only casein, and $P_{\text {prot }}$ approximated to the value of 0.02 (Gottwald et al., 2005; Gianferri et al., 2007a), a rough estimation of the value of $k_{\text {prot }}\left(\approx 6 \times 10^{3} \mathrm{~s}^{-1}\right)$ can be calculated (resulting in $k_{\text {prot }} \gg k_{d i f f}$ ). 
When $2 \mathrm{P}$ is taken into account $\left(T_{2,2 \mathrm{P}}^{-1} \approx 27 \mathrm{~s}^{-1}\right)$, the system is in the diffusion-limited relaxation regimen, in which the casein network can be considered as a surface relaxation sink.

In the diffusive-limited relaxation regimen, Eq. [3] (Belton et al., 1988; Gianferri et al., 2007a) applies:

$$
T_{2,2 \mathrm{P}}^{-1}=T_{2, w}^{-1}+\frac{\pi^{2} \cdot D}{a^{2}},
$$

assuming that the relaxation on the surface is fast compared with the diffusion (Belton et al., 1988). From Eq. [3], a maximum diffusion distance value of $a=22 \mu \mathrm{m}$ can be determined (Belton et al., 1988; Gianferri et al., 2007a). Thus, the characteristic dimension of spatial heterogeneity for the $2 \mathrm{P}$ compartment is in the range 4 $\mu \mathrm{m}<a<22 \mu \mathrm{m}$, in accordance with the value of $a=$ $17 \mu \mathrm{m}$, determined by calculating the average distance $(6 \mathrm{D} t)^{1 / 2}$ that a freely diffusing water molecule travels in a time $t$ equal to $T_{2, w}$ in a unrestricted diffusion regimen (Belton et al., 1988).

In summary, the data calculated in this work are in agreement with the values obtained in dried milk suspensions (Hills et al., 1990), in Mozzarella di Bufala Campana cheese (Gianferri et al., 2007b), and with other model systems (Belton et al., 1988; Hills et al., 1989). In particular, the similarity of $T_{2}$ distributions found in the present work and those determined in Mozzarella cheese (Gianferri et al., 2007a,b) and in other soft and hard cheeses (Chaland et al., 2000) suggests that cheese structure at molecular level is not drastically changed by the specific technological dairy process, and, consequently, that proton populations observed in this work can be reasonably attributed to 2 different compartments: one including a population of water molecules tightly entrapped within casein network that strongly interact with protein protons $(1 \mathrm{P})$, and the other with longer $T_{2}$ values due to water inside the casein network $(2 \mathrm{P})$. The average spatial heterogeneity can be roughly calculated to be in the range from approximately 5 to $25 \mu \mathrm{m}$.

Finally, we must address the possible influence of fat signal suppression on the water proton relaxation populations observed (Tables 1 and 2). First, because of the considerations discussed above and reported in Table 3, fat saturation had the effect of reducing $2 \mathrm{P}$ area fraction (of nonsaturated images) for both Fiore Sardo PDO and pecorino cheeses to an extent that depended on fat content. Second, because a selective pulse was applied at a frequency offset of about 1,050 $\mathrm{Hz}$ to suppress the fat signal, a portion of immobile protein protons pool (that have resonance frequency spread approximately 500 to $2,500 \mathrm{~Hz}$ away from bulk water) is also saturated, likely leading to an incidental magnetization transfer effect. This effect is well known and characterized, even if the exact mechanism by which transfer of magnetization from macromolecules protons to "free" water protons pool occurs (chemical exchange, dipole-dipole interactions) is somewhat debatable (Henkelman et al., 2001). The magnetization transfer effect may result in a decreased signal from mobile water, because of the exchange of magnetization between the "macromolecules" and "free" proton pools. In cheese, signal decrease is expected to be more relevant for the $1 \mathrm{P}$ water molecules that strongly interact, by means of chemical exchange, with labile protein protons (Grossman et al., 1994). The possible magnetization transfer effect should be more intense in HT cheeses, which always exhibited a more populated $1 \mathrm{P}$ compartment even without saturation (see Supplementary Data; http://dx.doi.org/10.3168/jds.2013-6607). Because 1P water protons are the most affected by interactions with macromolecular protons, the $1 \mathrm{P}$ area fraction was expected to be reduced by off-resonance pulses (fat saturation). Nevertheless, the 1P area fraction in HT milk cheese remained significantly higher than that in the raw milk counterparts (Tables 1 and 2 ). This suggests that the likely magnetization transfer effect did not affect the suitability of the MRI protocol for discrimination between raw milk Fiore Sardo and HT pecorino cheeses.

\section{CONCLUSIONS}

We developed a rapid, nondestructive, and robust procedure for discriminating Sardinian sheep milk cheese made from HT milk (pecorino cheese) and that made from raw milk (Fiore Sardo PDO cheese), by combining MRI measurement with multi-exponential analysis of water proton magnetization decay. The MRI procedure proposed was able to differentiate samples through proton $T_{2}$ distribution. In particular, a significantly higher area fraction corresponding to the population of water protons strongly interacting with proteins (1P) was observed in HT milk cheese compared with raw milk counterparts and the opposite was observed for the long $T_{2}$ population (2P). The MRI analysis described here can be carried out at room temperature and requires 6 to 18 min of experimental time. No complex data treatments are needed because appropriate software is available for this purpose. The results obtained on 36 cheese samples were in agreement with previously published data on similar systems. The proposed MRI protocol, with some improvements, offers several suggestions for future applications. For example, the MRI approach described could be performed simultaneously on different regions of interest within the whole cheese wheel 
(i.e., from the rind to the cheese paste center) to assess cheese microstructure and morphology together with the multi-exponential decay of magnetization curves. Further studies are required to provide a deeper description of molecular events or structures that determine the variation of the observed relaxometric parameters. Nonetheless, the MRI approach described is potentially a promising tool for evaluating heat treatment of milk in other types of cheese.

\section{ACKNOWLEDGMENTS}

This study was a part of the project "Biotecnologie applicate all'agroalimentare e alla zootecnia," funded by Sardinia Region government by means of Sardegna Ricerche Technology Park. The authors are grateful to the Consortium for the Protection of Fiore Sardo Cheese (Consorzio per la Tutela del Formaggio Fiore Sardo) for the fruitful collaboration and for providing cheese samples. Dario Longo from the Department of Chemistry and Molecular Imaging Center (University of Turin, Italy) is greatly acknowledged for helpful discussions on MRI data treatment.

\section{REFERENCES}

Anastasiou, A., and L. D. Hall. 2004. Optimisation of $T_{2}$ and $M_{0}$ measurements of bi-exponential systems. Magn. Reson. Imaging $22: 67-80$.

AOAC International. 2000. Official Methods of Analysis. 17th ed. AOAC International, Arlington, VA.

Bàrcenas, P., F. J. P. Elortondo, M. Albisu, J. Mège, L. B. Roseiro, M. F. Scintu, P. Torre, S. Loygorri, and P. Lavanchy. 2007. An international ring trial for the sensory evaluation of raw ewe milk cheese texture. Int. Dairy J. 17:1139-1146.

Belton, P. S., B. P. Hills, and E. R. Raimbaud. 1988. The effects of morphology and exchange on proton NMR relaxation in agarose gels. Mol. Phys. 63:825-842.

Bjarnason, T. A., and J. R. Mitchell. 2010. AnalyzeNNLS: Magnetic resonance multiexponential decay image analysis. J. Magn. Reson. 206:200-204

Budiman, M., R. L. Stroshine, and P. Cornillon. 2002. Moisture measurement in cheese analogue using stretched and multi-exponential models of the magnetic resonance $\mathrm{T}_{2}$ relaxation curve. J. Dairy Res. 69:619-632.

Buffa, M. N., A. J. Trujillo, M. Pavia, and B. Guamis. 2001. Changes in textural, microstructural, and colour characteristics during ripening of cheeses made from raw, pasteurized or high-pressuretreated goats' milk. Int. Dairy J. 11:927-934.

Caravan, P. 2006. Strategies for increasing the sensitivity of gadolinium based MRI contrast agents. Chem. Soc. Rev. 35:512-523.

Castell-Palou, A., C. Rosselló, A. Femenia, and S. Simal. 2012. Simultaneous quantification of fat and water content in cheese by TD-NMR. Food Bioprocess. Technol. 6:2685-2694. http://dx.doi. org/10.1007/s11947-012-0912-8.

Chaland, B., F. Mariette, P. Marchal, and J. De Certaines. 2000. ${ }^{1} \mathrm{H}$ nuclear magnetic resonance relaxometric characterization of fat and water states in soft and hard cheese. J. Dairy Res. 67:609618

Does, M. D., and R. E. Snyder. 1995. T2 relaxation of peripheral nerve measured in vivo. Magn. Reson. Imaging 13:575-580.
Dula, A. N., D. F. Gochberg, and M. D. Does. 2009. Optimal echo spacing for multi-echo imaging measurements of bi-exponential T2 relaxation. J. Magn. Reson. 196:149-156.

Everett, D. W., and M. A. E. Auty. 2008. Cheese structure and current methods of analysis. Int. Dairy J. 18:759-773.

Everett, D. W., and N. F. Olson. 2003. Free oil and rheology of Cheddar cheese containing fat globules stabilized with different proteins. J. Dairy Sci. 86:755-763.

Fenrich, F. R. E., C. Beaulieu, and P. S. Allen. 2001. Relaxation times and microstructures. NMR Biomed. 14:133-139.

Folch, J., M. Lees, and G. H. Sloane Stanley. 1957. A simple method for the isolation and purification of total lipids from animal tissues. J. Biol. Chem. 226:497-509.

Gianferri, R., V. D'Aiuto, R. Curini, M. Delfini, and E. Brosio. 2007a. Proton NMR transverse relaxation measurements to study water dynamic states and age-related changes in Mozzarella di Bufala Campana cheese. Food Chem. 105:720-726.

Gianferri, R., M. Maioli, M. Delfini, and E. Brosio. 2007b. A lowresolution and high-resolution nuclear magnetic resonance integrated approach to investigate the physical structure and metabolic profile of Mozzarella di Bufala Campana cheese. Int. Dairy J. $17: 167-176$.

Gonzalez Viñas, M. A., C. Ballesteros, P. J. Martín-Alvarez, and L. Cabezas. 2007. Relationship between sensory and instrumental measurements of texture for artisanal and industrial Manchego cheeses. J. Sens. Stud. 22:462-476.

Gottwald, A., L. K. Creamer, P. L. Hubbard, and P. T. Callaghan. 2005. Diffusion, relaxation, and chemical exchange in casein gels: A nuclear magnetic resonance study . J. Chem. Phys. 122:3450634516 .

Graham, S. J., P. L. Stanchev, and M. J. Bronskill. 1996. Criteria for analysis of multicomponent tissue $T_{2}$ relaxation data. Magn. Reson. Med. 35:370-378.

Grossman, R. I., J. M. Gomori, K. N. Ramer, F. J. Lexa, and M. D. Schnall. 1994. Magnetization transfer: Theory and clinical applications in neuroradiology. Radiographics 14:279-290.

Henkelman, R. M., G. J. Stanisz, and S. J. Graham. 2001. Magnetization transfer in MRI: A review. NMR Biomed. 14:57-64.

Hills, B. P., S. F. Takacs, and P. S. Belton. 1989. The effects of proteins on the proton N.M.R. transverse relaxation time of water. Mol. Phys. 67:919-937.

Hills, B. P., S. F. Takacs, and P. S. Belton. 1990. A new interpretation of proton NMR relaxation time: Measurements of water in food. Food Chem. 37:95-111.

Hirvi, Y., and M. W. Griffiths. 1998. Milk catalase activity as an indicator of thermization treatments. J. Dairy Sci. 81:338-345.

Kelly, A. L., N. Datta, and H. C. Deeth. 2006. Thermal processing of dairy products. Pages 265-298 in Thermal Food Processing: New Technology and Quality Issues. Vol. 1. D.-W. Sun, ed. CRC Press Taylor \& Francis Group, Boca Raton, FL.

Kuo, M. I., M. E. Anderson, and S. Gunasekaran. 2003. Determining effects of freezing on pasta filata and non pasta filata Mozzarella cheeses by nuclear magnetic resonance imaging. J. Dairy Sci. 86:2525-2536.

Kuo, M. I., S. Gunasekaran, M. Johnson, and C. Chen. 2001. Nuclear magnetic resonance study of water mobility in pasta filata and non pasta filata Mozzarella. J. Dairy Sci. 84:1950-1958.

Laule, C., I. M. Vavasour, S. H. Kolind, D. K. B. Li, T. L. Traboulsee, G. R. W. Moore, and A. L. MacKay. 2007. Magnetic resonance imaging of myelin. Neurotherapeutics 4:460-484.

Little, C. L., J. R. Rhoades, S. K. Sagoo, J. Harris, M. Greenwood, V. Mithani, K. Grant, and J. McLauchlin. 2008. Microbiological quality of retail cheeses made from thermized or pasteurized milk in the UK. Food Microbiol. 25:304-312.

MacKay, A., K. Whittall, J. Adler, D. Li, D. Paty, and D. Graeb. 1994. In vivo visualization of myelin water in brain by magnetic resonance. Magn. Reson. Med. 31:673-677.

Mahdjoub, R., J. Molegnana, M. J. Seurin, and A. Briguet. 2003. High resolution magnetic resonance imaging evaluation of cheese. J. Food Sci. 68:1982-1984. 
Mariette, F. 2006a. NMR relaxation of dairy products. Pages 16971701 in Modern Magnetic Resonance. Part III. G. A Webb, ed. Springer, Dordrecht, the Netherlands.

Mariette, F. 2006b. NMR imaging of dairy products. Pages 1801-1806 in Modern Magnetic Resonance. G. A. Webb, ed. Springer, Dordrecht, the Netherlands.

McCreary, C. R., T. A. Bjarnason, V. Skihar, J. R. Mitchell, V. W. Yong, and J. F. Dunn. 2009. Multiexponential $T_{2}$ and magnetization transfer MRI of demyelination and remyelination in murine spinal cord. Neuroimage 45:1173-1182.

Mendia, C., F. C. Ibanez, P. Torre, and Y. Barcina. 1999. Effect of pasteurization on the sensory characteristics of a ewe's-milk cheese. J. Sens. Stud. 14:415-424.

Métais, A., and F. Mariette. 2003. Determination of water self-diffusion coefficient in complex food products by low field ${ }^{1} \mathrm{H}$ PFG-NMR: Comparison between the standard spin-echo sequence and the $T_{1^{-}}$ weighted spin-echo sequence. J. Magn. Reson. 165:265-275.

Morales-Celaya, M. F., C. Lobato-Calleros, J. Alvarez-Ramirez, and E. J. Vernon-Carter. 2012. Effect of milk pasteurization and acidification method on the chemical composition and microstructure of a Mexican pasta filata cheese. Lebenson. Wiss. Technol. 45:132-141.

Noronha, N., E. Duggan, G. R. Ziegler, E. D. O'Riordan, and M. O'Sullivan. 2008. Investigation of imitation cheese matrix development using light microscopy and NMR relaxometry. Int. Dairy J. 18:641-648.

Piga, C., R. Di Salvo, and M. F. Scintu. 2012. Effetto dei blandi trattamenti termici sulle caratteristiche sensoriali dei formaggi ovine. $\mathrm{IV}^{\circ}$ Convegno Nazionale Società Italiana di Scienze Sensoriali, Trieste, Italy.
Pinto, G., S. Caira, M. Cuollo, O. Fierro, M. A. Nicolai, L. Chianese, and F. Addeo. 2012. Lactosylated casein phosphopeptides as specific indicators of heated milks. Anal. Bioanal. Chem. 402:19611972.

Polychroniadou, A. 2001. Eyes in cheese: A concise review. Milchwissenschaft 56:74-77.

Ramos-Cabrer, P., J. P. Van Duynhoven, H. Timmer, and K. Nicolay. 2006. Monitoring of moisture redistribution in multicomponent food systems by use of magnetic resonance imaging. J. Agric. Food Chem. 54:672-677.

Ruan, R., K. Chang, P. L. Chen, R. G. Fulcher, and E. D. Bastian. 1998. A magnetic resonance imaging technique for quantitative mapping of moisture and fat in a cheese block. J. Dairy Sci. 80:9-15.

Salvadori del Prato, O. 1998. Trattato di Tecnologia Casearia. Edagricole-Edizioni Agricole della Calderini s.r.l., Bologna, Italy.

Samelis, J., A. Lianou, A. Kakouri, C. Delbes, I. Rogelj, B. BogovicMatijasic, and M. C. Montel. 2009. Changes in the microbial composition of raw milk induced by thermization treatments applied prior to traditional Greek hard cheese processing. J. Food Prot. 72:783-790.

Scintu, M. F., A. Del Caro, P. P. Urgeghe, C. Piga, and R. Di Salvo. 2010. Sensory profile development for an Italian PDO ewe's milk cheese at two different ripening times. J. Sens. Stud. 25:577-590.

Whittall, K. P., A. L. MacKay, D. A. Graeb, R. A. Nugent, D. K. B. $\mathrm{Li}$, and D. W. Paty. 1997. In vivo measurement of $T_{2}$ distributions and water contents in normal human brain. Magn. Reson. Med. $37: 34-43$. 